\title{
DESIGN DAYS (RE)BOOT CAMP: INTEGRATING FIRST-YEAR ENGINEERING DESIGN REMOTELY
}

\author{
Jennifer Howcroft, Igor Ivkovic*, Matt. Borland, Reem Roufail, and Carolyn MacGregor \\ Systems Design Engineering, University of Waterloo \\ *published posthumously \\ jenny.howcroft@uwaterloo.ca, igor.ivkovic@uwaterloo.ca, mjborlan@uwaterloo.ca, rroufail@uwaterloo.ca, \\ cgmacgre@uwaterloo.ca
}

\begin{abstract}
Due to the COVID-19 pandemic, course activities pivoted to online learning in 2020. This necessitated a redesign of Systems Design Engineering's Design Days from a two-day in-person event at the start of term to three shorter online sessions throughout term to prepare and support students during their first term at university. These sessions featured new activities including, an arcade game developed using block coding; a Rube Goldberg virtual ball toss; and the design of a WiFi access point reflector. All activities were designed using principles of effective online learning including, social presence, familiarization with online learning technology, and engaging activities. The online Design Days (Re)Boot Camp ran successfully during Fall 2020, supporting most intended learning outcomes and achieving high initial participation. Continual improvement will focus on sustaining participation, strengthening connections to design and technical concepts, and retaining strong elements upon the return to in-person learning.
\end{abstract}

Keywords: Engineering design, Remote delivery, Online education, Intended learning objectives, First year students, Iterative design

\section{INTRODUCTION}

Prior to Fall 2020, the Department of Systems Design Engineering (SYDE) introduced its approximately 200 incoming first-year students to their engineering design course sequence through an intensive two-day Design Days Boot Camp [1], [2]. The on-campus Design Days Boot Camp provided an opportunity for students to experience collaborative problem-based learning, engage in hands-on engineering design experiences, and begin building relationships with their peers, departmental staff and faculty. The Design Days activities instantiated the nine intended learning outcomes (ILOs) presented in [2] and further discussed in Section 3.1. SYDE's on-campus Design Days Boot Camp has become a critical training component for a successful term and prepares the first-year students to engage in real-world design problems in their first-semester design course [1], [2].

In Fall 2020, the COVID-19 pandemic necessitated a redesign of the Design Days Boot Camp from an oncampus, in-person two-day event to a new format more appropriate for online, remote learning. A successful redesign required careful consideration of effective online learning principles and guidelines.

While many online learning considerations and instructional design principles exist, to the best of our knowledge, a clear consensus on the optimal strategy for teaching engineering online is lacking. To increase the likelihood that the Design Days Boot Camp online activities would be effective for first year students a subset of instructional design guiding principles was selected: social presence [3]-[6], familiarization with online learning technology [4], [7], and engaging activities [5], [7]. Other principles mentioned in the literature include, in no particular order, cost effectiveness, access, and usefulness of technology [3], [7], [8]; student and faculty satisfaction [3]; safe learning environments [4]; and cooperative and collaborative learning [5], [7].

Held in the first days of the first term, Design Days Boot Camp represents the students' first formal interactions with one another and with faculty and staff of the department. Early interactions to build a sense of social presence should support collaboration between students and student comfort in reaching out to instructors throughout the term. Familiarization with online learning technology was prioritized as students would be expected to access learning materials throughout the term using university-supported platforms that may be new to students entering the university (e.g., Bongo® - Virtual Classroom [9] and Microsoft (MS) Teams ${ }^{\circledR}$ [10]). The first Design Days (Re)Boot Camp session presented an opportunity for students to use the learning platforms in a low-stakes manner. Finally, the guiding principle of engaging activities aligned well with the pre-existing approach to Design Days Boot Camp, as a hands-on approach to introduce engineering design and teamwork. 
The paper is structured as follows: Section 2.0 presents the redesigned, online Design Days format, timeline, and associated activities. Section 3.0 discusses the activities with respect to the original ILOs and the three selected instructional design principles. Section 4.0 presents reflections on student engagement with the online Design Days format Section 5.0 presents the tentative plan looking towards Fall 2021, with overall conclusions in Section 6.0.

\section{DESIGN DAYS (RE)BOOT CAMP}

\subsection{Online Format Overview \& Timeline}

The on-campus format of two full days of activities during the first two days of term was not feasible for an online delivery of Design Days, as it is unlikely that students would remain engaged over an eight-hour period. Thus, Design Days (Re) Boot Camp was divided into three smaller sessions throughout the semester as shown in Fig. 1 .

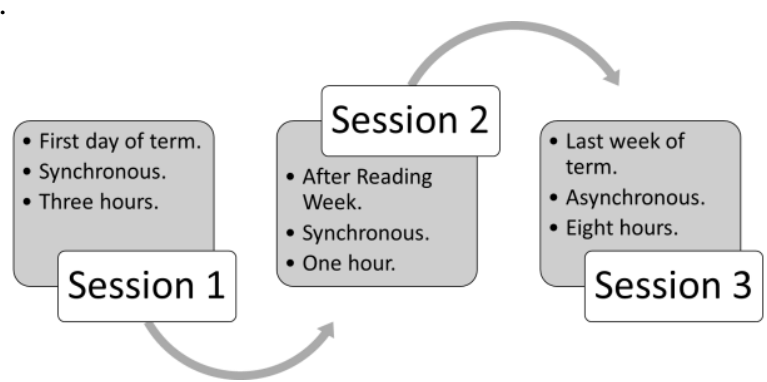

Figure 1. Timeline of Design Days (Re)Boot Camp.

In previous years, Design Days was run concurrently for both systems design engineering (SYDE) and biomedical engineering (BME) first-year students ( 200 students total). The concurrent format provided an interdisciplinary aspect to the activities with teams consisting of students from both cohorts. Decisions were made for the online format to be delivered separately to each program- sections ( $\sim 100$ students per section) due to the limits on participant enrollment associated with the various online learning platform technologies (e.g., Bongo ${ }^{\circledR}$ - Virtual Classroom has a limit of 150 students, MS Teams ${ }^{\circledR}$ has a limit of 30 private channels per group). The sessions run for SYDE and BME cohorts were essentially the same.

All synchronous sessions were recorded so that they could be viewed asynchronously. Students unable to attend synchronously due to time zones, schedule conflicts, or other reasons could still access session content and still engage in at least a marginal manner.

2.1.1. Session 1. Session 1 (S1) was held on the first day of term. It was a synchronous, recorded session that lasted three hours. In this session, students were provided with opportunities to become familiar with the online learning platforms to be used throughout the term. As a mandatory ungraded orientation event, students were strongly encouraged to attend. While student attendance was not formally tracked, participation in the synchronous session was between 95 and $100 \%$. S1 included several activities to foster social presence and engagement discussed in more detail in Section 2.2.1.

2.1.2. Session 2. Session 2 (S2) was an optional session offered during the middle of term, after the fall Reading Week. S2 was a synchronous, recorded session lasting one hour with focus on a single engaging activity to support ongoing social presence through student-student and studentfaculty interactions. Participation in the S2 synchronous session was between 25 and 30\%. The Rube Goldberg virtual toss activity is described in Section 2.2.2.

2.1.3. Session 3. Session 3 (S3) occurred during the last week of term and was integrated into the first-semester design course. The intention of this learning activity was to provide a hands-on, engaging way to experience the importance of testing in the design process. S3 centered around a graded, self-paced, individual activity supported by written instructions and instructor support with expectations that students would take approximately eight hours to complete all components spread over a one-week period in lieu of other design course hours. The Wi-Fi access point reflector activity is described in Section 2.2.3.

In late fall 2020, our community experienced the unexpected passing of Igor Ivkovic, one of the first-year instructors for both SYDE and BME cohorts and a coauthor of this paper. To allow colleagues and students space and time to grieve, S3 was made optional. Participation in S3, based on the number of submitted design reports, was understandably low $(<5 \%)$.

\subsection{Design Days Activities for each Session}

2.2.1. Session 1 (S1) Activities. S1 consisted of several activities that occurred over the three-hour synchronous session including, introduction to the department, Kahoot!® department trivia quiz, an ice breaker activity, a software activity, a calculator toss activity, and a session wrap-up. Connections between these activities and ILOs will be addressed in Section 3.

The introduction to the department included welcomes by the department chair and associated chair of undergraduate studies for the systems design engineering students or the biomedical engineering program director for the biomedical engineering students. Additional introductions included some of their first-term professors, key administrative and technical staff, brief presentation on department history, and an overview of their program. The synchronous nature of these welcomes was chosen to strengthen social presence to increase the likelihood that students would feel comfortable reaching out to these department members later in the term if needed.

After the welcome, to foster social presence and engagement, students participated in a Kahoot!® [11] department trivia quiz. Questions in the quiz focused on 
the department and program information presented in the welcome, and prizes were given to top scoring students.

Students were then introduced to MS Teams®, used as an online learning platform at the University of Waterloo. Students had been pre-assigned to teams of five or six and private channels for these teams had been set-up in a Design Days group on MS Teams ${ }^{\circledR}$ (24 teams for the SYDE cohort and 26 teams for the BME cohort). Once logged into MS Teams ${ }^{\circledR}$, students met synchronously to complete an ice-breaker team-building activity with their teams. This activity was a fill-in-the-blanks activity, similar to Mad Libs ${ }^{\mathrm{TM}}$ word game, where students collaboratively created a description of a future engineer in their program. The completed description was posted to the general, public channel in the Design Days group for everyone to read on MS Teams.

After a short break, students worked with the same teams to complete a software activity using Microsoft's open source MakeCode [12] coding platform that is suitable for beginners. With MakeCode Arcade students created an arcade game similar to the Cyclone arcade game [13]. See Fig. 2. A scaffolding approach to programming was used in that the instructors provided students with detailed instructions and tips for using block coding techniques. An example of a final game interface is shown in Fig. 3. Faculty and graduate students were available to provide support and answer questions throughout this activity through chat-based or video-based interactions.

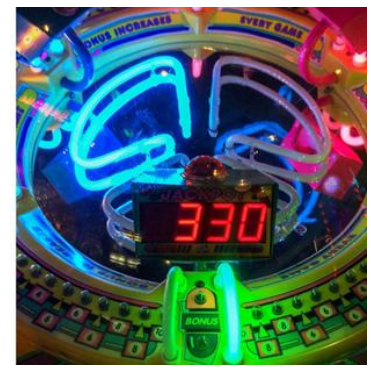

Figure 2. Example of a Cyclone Arcade game [13].

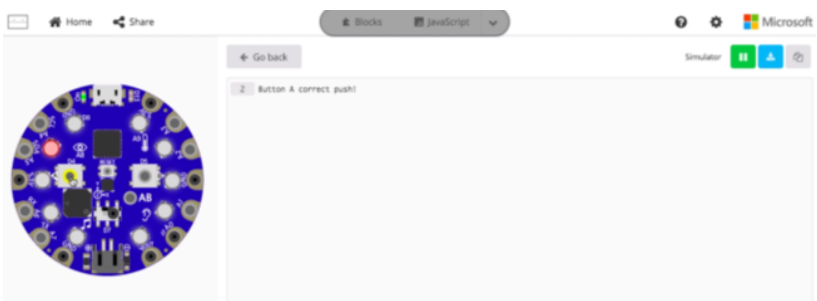

Figure 3. Example of a final arcade game interface.

Next students engaged in a shorter activity inspired by the "toilet paper toss" meme videos [14] that were popular on social media during the beginning of the pandemic. Students were tasked with completing a virtual calculator toss with their entire team during a recorded meeting session and sharing the recorded video on the general, public channel in the Design Days group for everyone to watch on MS Teams. Based on available viewing data, each team calculator toss video was viewed between nine and fifteen times. All videos were viewed and liked by the instructional team to foster social presence and select cohort winners for best arcade game.

A brief closing session was held for students to reflect on the session's activities, get information on prizes to come, and receive congratulations on their great work throughout the session.

2.2.2. Session 2 (S2) Activity. S2 centered around one activity: a Rube Goldberg virtual ball toss that increased the challenge of the calculator toss included in S1. In this one-hour session, students worked with the same teams from the S1 to create a Rube Goldberg Machine that would pass a ball (or any round object) virtually between all team members. Students were not allowed to directly touch the ball but could make indirect contact through a crank, lever, or other similar simple machine, physics-inspired interaction points. Students were reminded of the iterative design process they were learning in their first-term design courses. This activity focused on ideation, prototyping, and testing with strong encouragement to iterate as needed throughout the session. Students also received a mini lesson on the conservation of energy, potential energy, kinetic energy, and work to help them succeed in this task. Similar to the calculator toss, students recorded a video meeting of their virtual ball toss and shared this recording on the general, public channel in the Design Days group for everyone to watch on MS Teams. Based on available viewing data, each Rube Goldberg Machine video was viewed between twelve and twenty-two times. All videos were viewed and liked by the instructional team to encourage social presence, and winning teams were selected for each cohort. High-level feedback regarding the activities was to be provided at the end of term when winning teams were announced, but this did not occur in large part due to the passing of Igor Ivkovic, a key member of the Design Days team and author on this paper.

2.2.3. Session 3 (S3) Activity. S3 also centered around one activity: designing and testing a Wi-Fi access point reflector. The third session was integrated into the firstterm design courses for both the SYDE and BME first-year students. It was a graded deliverable during the final week of term. There were no other deliverables, lectures, or other course activities occurring that week for the design course in which the activity was embedded. Due to the hands-on nature of this activity, with physical prototyping and testing, and public health guidance related to the COVID19 pandemic, this was an individual student activity.

This activity was inspired by a real-world problem during the COVID-19 pandemic that being the increased prevalence of work-from-home and study-from-home occurring during the pandemic and the increased load these activities are placing on the internet infrastructure, 
including Wi-Fi access points. Real world relevance can increase student engagement, one of the guiding principles, and is essential in a professional engineering program. Real world applications show students the relevance of activities to their future careers and the broader world. Successfully completing this activity had the potential to improve internet quality for students if they were experiencing issues.

To start the activity, students were provided the following situation impact statement:

'Design a Wi-Fi access point (e.g., Wi-Fi router)

reflector to be used by students to improve Wi-Fi

range and reception in their homes, that improves

Wi-Fi signal strength by $6 \mathrm{dBm}$ and achieves a

minimum signal strength of $-67 \mathrm{dBm}$ in front of the

reflector and Wi-Fi access point.'

As well as a short situation of concern paragraph, constraints, and a requirement. The provided requirement was 'should increase Wi-Fi signal strength' and included a testing protocol and metric. The metric included a benchmark signal strength of $-67 \mathrm{dBm}$ and signal increase of $6 \mathrm{dBm}$ improvement. Constraints included dimensional restrictions, cost restrictions, and prohibition of direct contact between the design and Wi-Fi access point.

Students were expected to create two reflector design ideas that would increase signal strength $(\sim 1 \mathrm{hr})$, create two low fidelity prototypes of their ideas that could be tested ( $\sim 1$ to $2 \mathrm{hrs}$ ), and test their prototypes to assess their ability to increase Wi-Fi signal strength ( $\sim$ to $2 \mathrm{hrs})$. After completing these design activities, they needed to present their work in a five-page design report ( 2 to $3 \mathrm{hrs}$ ). The whole activity was intended to take up to eight hours to complete, the expected weekly workload for one course, with work spread throughout one week. No other course activities occurred during the design course that week.

To facilitate task completion and support students, detailed written instructions were provided and supplemented with a synchronous, recorded instruction session. Course instructors were also available throughout the week to answer questions, clarify expectations, and meet with students on an as-needed basis through MS Teams ${ }^{\circledR}$ in chat-based or video-based interactions.

\section{INSTRUCTIONAL GOALS, ILOS AND ONLINE TEACHING PRINCIPLES}

Using the instructional design guiding principles of social presence, familiarization with online learning technology, the online Design Days (Re)Boot Camp was designed to support as many of the original Design Days ILOs as possible while also striving for a positive online learning experience. In the end, 8 of the original 9 ILOs were integrated into the redesigned Design Days (Re)Boot Camp through activities in the different sessions and one additional, online-learning-specific ILO was added. However, a lack of assessment of these ILOs means that it is unclear whether these were fully realized in terms of actual student learning. Meaningfully measuring student performance on these ILOs without adding to student workload remain an area of future work.

\subsection{Supporting ILOs}

3.1.1. Demonstrating an understanding of their program of study and related courses, resources, faculty and teaching assistants, and traditions within the department or faculty. This was predominantly accomplished during S1 with the welcome by key faculty, introduction to key administrative and technical staff, and introductions by some first-term instructors. In addition, students were introduced to the department and chosen program during the welcome and follow-up department trivia activity. Students demonstrated their acquired knowledge through the Kahoot!@ department quiz and fillin-the-blanks activity. While the fill-in-the-blanks activity focused more on 'fun' entries than those demonstrating 'acquired knowledge', the Kahoot!@ quiz did show some evidence of short-term knowledge acquisition although specific performance data (i.e., percent correct responses) is not available. Finally, faculty and teaching assistants were available through MS Teams ${ }^{\circledR}$ and a virtual classroom to provide support throughout all sessions ( $\mathrm{S}$, S2, and S3). Connections were made between the software activity and the first-term software course, between the design activities and first-term design course, and between the ball toss activity and a physics (dynamics) course.

3.1.2. Showing a sense of community within their program, the department, and the faculty. The dedicated MS Teams® Design Days groups provided an opportunity for community building throughout all the sessions (S1, S2, and S3). Students were able to connect with their classmates and team members through the general and private channels, respectively. Additionally, student teams shared their team-building engineering student description, calculator toss video, and ball toss videos in the general chat. Video viewing data available indicates videos were viewed between nine and twenty-two times by instructors and students. Instructors made a point of liking posts and providing some individual comments. This encouraged students to do the same, although only a few students $(<5)$ tended to do this on each individual post.

3.1.3. Demonstrating an understanding of how design and technical courses are related as part of the program. The software activity in S1 was connected to BME/SYDE121 - Digital Computation as the course pedagogy make use of block coding and pair programming. Additionally, the Rube Goldberg ball toss activity during the second session (S2) was connected to BME/SYDE182 - Physics 2 - Dynamics. Students were introduced to conservation of energy concepts including kinetic energy, potential energy, and work that are formally taught in this course. All design activities were connected to 
BME/SYDE161 - the first semester design course and the final activity was fully integrated into this course. Design course instructors report that they often asked students about previous experience with design-related concepts or tasks, like prototyping. Frequent responses included recalled Design Days experiences, demonstrating awareness of connections between activities and courses.

3.1.4. Explaining the importance of safety during design activities and recognize important safety standards. This ILO was met at an introductory level during all sessions (S1, S2, and S3). Students were reminded of the importance of safety during the calculator toss (S1) and ball toss (S2). Additionally, a safety-focused constraint was included in the design of the Wi-Fi access point reflector: students were prohibited from having their device touch the Wi-Fi access point or from modifying the Wi-Fi access point in any way. The integration of safety into all sessions was focused on responsible prototyping and testing practices. While safety knowledge was not directly measured, no unsafe practices were observed in any of the video recordings or other student submissions and no injuries were reported.

3.1.5. Using common machining tools in a machine shop setting. This ILO was removed for the online Design Days (Re)Boot Camp due to the necessity of all activities being delivered remotely. It was not possible for students to safely access the machine shop.

3.1.6. Understand a design problem before attempting to create a solution. This ILO was focused on the later stages of the design process (i.e., ideation, prototyping, and testing). There were two main design activities during Design Days (Re)Boot Camp: the Rube Goldberg ball toss (S2) and the design and testing of the Wi-Fi access point reflector (S3). Both activities would be more successfully accomplished if students took the time to understand the problem before solving. For the Rube Goldberg ball toss, successful completion would be demonstrated through a recorded video of a virtual ball toss involving no direct contact with the ball. All participating teams met this success criterion. For the Wi-Fi access point reflector, successful completion would be designing a reflector that met the requirement benchmark of $6 \mathrm{dBm} \mathrm{Wi}-\mathrm{Fi}$ signal strength improvement. All participating students met this success criterion. As part of mentoring, students were given guided instructions. For the Rube Goldberg ball toss, students were guided through the process of understanding the design problem via a synchronous, recorded session before engaging in building their design. The synchronous session included reminders of the iterative design process and how to apply it to the activity, relevant engineering concepts, and activity constraints. This gave all students a chance to consider the problem before creating a solution. For the Wi-Fi access point reflector, students were provided with written instructions that contained key information important to understanding the problem. This was presented using engineering design process terminology from their design course like situation impact statement, situation of concern, requirements, and constraints. A synchronous, recorded instruction session was provided early in the week to give students an opportunity to consider this information with the course instructor, clarify areas of uncertainty, and support the development of a clear understanding of the design problem before designing the reflector.

\subsubsection{Work and communicate in a team environment.} Throughout the first two sessions (S1, S2) students worked in teams of five or six students. Group communication and teamwork were conducted primarily through the MS Teams ${ }^{\circledR}$ private channels. It is possible that non-supported platforms were also used by students to facilitate group communications (e.g., Facebook Messenger ${ }^{\circledR}$, Discord $\AA$, Zoom ${ }^{\circledR}$, etc.). However, given their unsupported status, it is unknown if and how much these platforms were used. Activities dependent on successful teamwork include, the team-building activity, the software activity, the calculator toss, and the ball toss. Besides communicating with their team members, students communicated with faculty and teaching assistants to successfully achieve activity goals (S1, S2, and S3).

3.1.8. Follow a design process and apply iterative design towards the creation of a design solution. Similar to 3.1.6, an iterative design process was encouraged for both the Rube Goldberg ball toss (S2) and the design and testing of the Wi-Fi access point reflector (S3). Both activities focused on the later half of the design process due to time constraints: ideation, prototyping, and testing. While iteration was encouraged in these activities, it was not necessary to achieve success. That being said, iterations likely occurred in the S1 and S2 activities. In S1, some students re-recorded their calculator toss videos after realizing that recorded screen positions did not correspond with live meeting screen positions, leading to an ineffective virtual pass effect. In S2, there was some evidence of iterations in the recorded videos with students being heard asking questions like 'Do you want to try again?' or 'Do you think we got it this time?'. Iterations were more formally included in S3 where plans for future iterations were included as part of the written report.

\subsubsection{Test design solutions and interpret test results as} part of iterative design. This ILO was predominantly addressed in S3 through the design and testing of the Wi$\mathrm{Fi}$ access point reflector. In S3, students were provided with a test protocol that would allow assessment of the requirement 'should increase Wi-Fi signal strength'. The test protocol did provide some areas of flexibility where students needed to make appropriate choices to test their designs in their own home environments. These studentspecific choices needed to be clearly communicated in their 
design reports along with the test results and interpretation of those results. This was demonstrated in all the submitted reports where students communicated their customized test protocols, including device and measurement locations, that were appropriate for their home environments. Methods of communication often included diagrams or annotated pictures of their home environments with device and measurement locations clearly identified and communication of relevant distances. The timing of this activity at the end of the semester allowed for realistic testing to occur and an expectation for reasonable interpretation that would inform a future design iteration.

3.1.10. Use online learning technology. This ILO was newly added for online Design Days (Re)Boot Camp and was an important addition to prepare first-year students for a successful online term given that university-specific technologies likely differed from technologies used in their high school. Students were familiarized with two key online learning platforms: LEARN® and MS Teams®. This ILO was important for S1 as this session was when most students were exposed to these specific online platforms for the first time. Across the first session activities, students attended a synchronous lecture in the LEARN® virtual classroom, accessed and shared files through MS teams, posted to a general channel in an MS Teams ${ }^{\circledR}$ group, and participated in non-recorded and recorded team meetings in MS Teams®. These all represent key skills that were used throughout the semester, particularly in the first-term design courses where MS Teams ${ }^{\circledR}$ was used heavily for design work.

\subsection{Incorporating Principles of Online Learning}

3.2.1. Social Presence. Social presence was identified as important for online learning success [3]-[6] and involves opportunities for collaboration, connections between student peers, and connections between students and faculty. All the online Design Days (Re)Boot Camp activities were designed to facilitate at least one of these types of social presence with many designed to support all types. As stated in 3.1.7., collaboration through teamwork was needed to complete the team-building activity, the software activity, the calculator toss, and the ball toss. Connections between student peers were encouraged through teamwork, and through the MS Teams® Design Days group general channel (e.g., liking other team's creations, posting to this channel, etc.). Connections between students and faculty were supported through synchronous sessions associated with all activities where students could ask questions through chat windows in the LEARN® virtual classroom. Students could also engage with faculty while completing activities synchronously or asynchronously through the MS Teams® Design Days group (chat or video-based interactions). Faculty also actively engaged with students using the MS Teams ${ }^{\circledR}$ Design Days group by liking student posts, providing post- specific comments, and announcing Design Days award winners. Therefore, social presence was supported throughout online Design Days (Re)Boot Camp activities.

3.2.2. Familiarization with online learning technology. For students to successfully engage with online learning, they first need to be able to successfully engage with the online learning technologies. In fact, technological issues are often identified as a key barrier to online learning [15], [16]. Therefore, orienting and familiarizing students with online learning technologies was a new, important component of Design Days (Re)Boot Camp as reflected in the new ILO. The integration of this principle aligns with supporting the new ILO, which was described in 3.1.10.

3.2.3. Engaging activities. Student engagement is seen as critical to successful online learning [5]. The intentions of Design Days activities are to be engaging, encourage active learning, provide real world relevance, and align with student motivations to learn engineering relevant skills.

Active learning is defined as: 'a method of learning in which students are actively or experientially involved in the learning process and where there are different levels of active learning, depending on student involvement [17].' Even with the challenges of remote instruction, experiential learning opportunities were implemented through the software activity, Rube Goldberg ball toss activity, and design of the Wi-Fi access point reflector activity. Students worked to apply and advance their understanding of technical and design skills through these activities at a level appropriate for the timing of the activity throughout the first term.

Real world relevance can increase student engagement. As discussed in 2.2.3, the design of the Wi-Fi access point reflector activity was directly connected to the increased prevalence of work-from-home and study-from-home occurring during the pandemic and the increased load these activities are placing on the internet infrastructure, including $\mathrm{Wi}-\mathrm{Fi}$ access points. Our intention was to increase engagement by giving students a potentially useful outcome: improved internet quality for students, if they were experiencing issues. In practice, all students who completed this activity started with adequate Wi-Fi signal strength (i.e., they were not experiencing internet quality issues and did not need the designed reflector).

Finally, instructors connected online Design Days (Re)Boot Camp activities to student motivators including student connections and student success. Students typically enter university with a motivation to meet their classmates, as exemplified by university and student-driven orientation activities at the beginning of in-person fall terms. Enabling student connections is a particular challenge in online learning. Therefore, activities were designed to facilitate student connections. Similarly, students were motivated to be successful in their studies, therefore when appropriate, activities were connected to their studies (see 3.1.3.). 


\section{STUDENT ENGAGEMENT \& INSTRUCTOR REFLECTIONS}

While students were not formally surveyed about online Design Days (Re)Boot Camp due to time constraints, anecdotal evidence from instructors indicate that the redesigned Design Days was effective and appreciated by first year students in both programs. Students shared that the activities helped them familiarize themselves with online learning technologies used in their courses and provided an enjoyable way to meet and engage with their new classmates. Even though students reflected positively on their Design Days experiences, student engagement and participation with Design Days activities declined as the term progressed. Participation in the first session was high (95 to 100\%). Participation was moderate in the second session $(25$ to $30 \%)$ and low in the third session $(<5 \%)$. As previously mentioned, the third session was made optional due to the passing of Igor Ivkovic.

From an instructor perspective, Design Days played a notable role in the success of the first term design project teamwork. Students started their first-year design course with a good foundation in online learning technology skills, experience collaborating online, and an introduction to their program of study. Similar to previous years with on-campus Design Days activities, the first-year students started term ready to engage in a real-world design problem in the design course. Additionally, Design Days helped prepare students for their first software-focused course. A common issue with introductory programming courses is large differences in student exposure to computer programming at the beginning of the course. Some students enter engineering programs with extensive coding experience and others with minimal experience. The arcade game software activity was designed to create a friendly and inclusive environment for all levels of programmers. The types of instructional supports and tips used in the Design Days activities set the stage for the level of support that students could expect in their first programming course. The instructors hypothesize that the software activity provides students with an opportunity to selfassess behaviour, ask questions about fundamental coding statements like if clauses, while loops, etc., and boost confidence after completing a coding activity in a relatively short time frame. These instructor hypotheses need to be assessed with student-driven data regarding the student experience (e.g., student survey), which will be discussed as an area of future work in the next section.

Ultimately, Design Days is intended to start the process of building community within the cohort. In this COVIDaffected teaching environment the intentional development of community becomes even more important.

\section{LOOKING AHEAD}

At the time of writing this paper, it is unknown whether Fall 2021 course activities will be delivered predominantly online or on-campus. While the availability of vaccines for COVID-19 is encouraging, it is likely that aspects of online learning will continue as students and faculty return to campus. The online Design Days (Re)Boot Camp activities described in this paper were generally successful at providing a positive online learning experience, meeting instructional goals, and supporting the ILOs for students. Based on instructor input, minimal changes are anticipated to run these activities again in Fall 2021.

Modifications to improve another online offering of Design Days include:

1. Changing the ice-breaker team-building activity to something with a strong connection to design. This could include a forced connections activity [18] or other, similar rapid design ideation technique.

2. Moving session 2 to the week before Reading Week instead of after Reading Week. Holding the session earlier in term may increase participation to further social presence and community building prior to midterm tests and assignments which typically happen after Reading Week.

3. Strengthening the connection to physics concepts in the Rube Goldberg Ball Toss. This could be done by providing pre-recorded examples of the activity with examples of kinetic energy, potential energy, and work highlighted by the instructor; however, the potential danger of groupthink with this approach would need to be carefully considered. Additionally, physics-related feedback could be provided to students specific to their virtual ball toss videos.

In addition to these improvements to the online offering of Design Days, work is needed to improve the gathering of evidence and indicators of achievement of ILOs. With the additional demands of the pandemic, the focus was predominantly on achieving an online version and evidence gathering was given a lower priority. However, with a second offering, greater priority will be given to evidence gathering that could include, but not be limited to, surveying the students regarding their level of engagement, platform usage, acquired knowledge, etc.; more detailed tracking of MS Teams usage; and collecting Kahoot ${ }^{\circledR}$ quiz metrics.

Eventually most course activities will return to campus and in-person learning, including Design Days. Therefore, it is also important to consider which activities should be retained from the online version. Some activities that will likely be retained in the transition back to in-person instructions include:

1. The team-building ice-breaker activity (S1) will likely be retained for in-person instruction after implementing the improvements described above. The ice-breaker activity during the introduction and 
welcome session, serves a useful purpose of framing student expectations for a highly collaborative day during the introduction and welcome session.

2. Once Design Days transitions back to in-person, it will likely revert to a beginning of term, two-day format. It remains of interest to continue the S3 activity, designing and testing a Wi-Fi access point reflector, as a summative individual student assessment to replace a traditional final exam in first term design courses.

\section{CONCLUSION}

Design Days Boot Camp has been through several cycles of iteration and improvement over the years, but none were as drastic as the transition to online learning. The online Design Days (Re)Boot Camp moved from a two-day beginning of term event to three smaller sessions distributed throughout the term with activities aimed at the anticipated developing skill level of the students at progressive points in the term. In addition to targeting changing skill levels, the activities were designed with principles of effective online learning in mind including, social presence, familiarization with online learning technologies, and engaging activities. The online format is viewed as successfully with eight of the original nine ILOs supported and an additional ILO added specifically targeting online learning. Areas for continual improvement include increasing participation in later term activities and strengthening activity connections to design and technical concepts.

\section{Acknowledgements}

We would like to thank Thomas Willett and Maud Gorbet for their substantial contributions and involvement with the in-person iterations of Design Days Boot Camp. We would also like to thank the Department of Systems Design Engineering for their support of Design Days. Our coauthor, Dr. Igor Ivkovic, passed away unexpectedly on November 27, 2020. He had been involved in Design Days since its inception and played a critical role in its successful transition to an online format. Igor was a passionate instructor who inspired students and colleagues alike and will be greatly missed.

\section{References}

[1] I. Ivkovic, T. L. Willett, M. J. Borland, and M. Gorbet, "Design days boot camp: enhancing student motivation to start thinking in engineering design terms in the first year," 1,2017 , doi: 10.24908/pceea.v0i0.9759.

[2] J. Howcroft, I. Ivkovic, M. J. Borland, and M. Gorbet, "Design Days Boot Camp 2.0: Improvements and connections to CEAB graduate attributes," Proceedings of the Canadian Engineering Education
Association (CEEA), Oct. 2019, doi: 10.24908/pceea.vi0.13713.

[3] J. Bourne, D. Harris, and F. Mayadas, "Online Engineering Education: Learning Anywhere, Anytime," Journal of Engineering Education, vol. 94, no. 1, pp. 131-146, 2005, doi: https://doi.org/10.1002/j.2168-9830.2005.tb00834.x.

[4] H. G. Crawford-Ferre and L. R. Wiest, "Effective Online Instruction in Higher Education," Quarterly Review of Distance Education, vol. 13, no. 1, pp. 1114, 2012.

[5] M. D. Dixson, "Creating Effective Student Engagement in Online Courses: What Do Students Find Engaging?," Journal of the Scholarship of Teaching and Learning, vol. 10, no. 2, pp. 1-13, Jun. 2010.

[6] J. C. Richardson and K. Swan, "Examining social presence in online courses in relation to students' perceived learning and satisfaction," Online Learning, vol. 7, no. 1, Art. no. 1, Mar. 2019, doi: 10.24059/olj.v7i1.1864.

[7] J. Webster and P. Hackley, "Teaching Effectiveness in Technology-Mediated Distance Learning," The Academy of Management Journal, vol. 40, no. 6, pp. 1282-1309, 1997, doi: 10.2307/257034.

[8] T. Volery and D. Lord, "Critical success factors in online education," International Journal of Educational Management, vol. 14, no. 5, pp. 216-223, Jan. 2000, doi: 10.1108/09513540010344731.

[9] Bongo - Virtual Classroom. Bongo, 2020.

[10] "Microsoft Teams,"
https://www.microsoft.com/en-ca/microsoft-

2020. teams/group-chat-software.

[11] “Kahoot!," 2020. https://kahoot.com/schools/.

[12] "Microsoft $\quad$ MakeCode," 2020. https://www.microsoft.com/en-us/makecode.

[13] "ICE Game - Cyclone," ICE Game, 2021. https://www.icegame.com/category/93/cyclone (accessed Mar. 04, 2021).

[14] "The Great Pandemic Toilet Paper Toss," Apr. 15, 2020.

https://www.youtube.com/watch?v=H224D9Q8Ck\&ab_channel=KaiF (accessed Feb. 22, 2021).

[15] S. A. Lloyd, M. M. Byrne, and T. S. McCoy, "FacultyPerceived Barriers of Online Education," vol. 8, no. 1, p. 12, 2012.

[16] J. Keengwe and T. T. Kidd, "Towards Best Practices in Online Learning and Teaching in Higher Education," vol. 6, no. 2, p. 9, 2010.

[17] C. C. Bonwell and J. A. Eison, Active Learning: Creating Excitement in the Classroom. 1991 ASHEERIC Higher Education Reports. ERIC Clearinghouse on Higher Education, 1991.

[18] E. Lupton, Design is Storytelling. New York, NY: Cooper Hewitt, Smithsonian Design Museum. 